\title{
An index to evaluate the amount of the solar radiation for a surface with eight directions
}

\author{
Yuki Koyamatsu*, Atushi Shiota, Yasunori Mitani, Yaser Soliman Qudaih, \\ Kiyotaka Fuji
}

Department of Electrical and Electronic Engineering, Kyushu Institute of Technology, Kitakyushu,804-8550, Japan

\begin{abstract}
The utilization of renewable energy and solar energy in specific is rapidly increasing in Japan. This research, it is proposes a method to find the most efficient angle and direction for the solar radiation when the solar power is introduced by using Geographic Information System (GIS) in Kitakyushu city, Fukuoka Prefecture as an example. The results revealed that the south facing angle of 25 degrees is the most efficient angle on the solar power for Kitakyushu city, Fukuoka Prefecture, Japan. The results are promising and required for the best utilization of solar energy in Japan. The method is applicable anywhere in the world with the availability of GIS data access.
\end{abstract}

Keywords: Geographic Information System, Solar radiation, solar power

\section{Introduction}

Recently, there has been a growing focus on renewable energy as a solution for the aggravating fossil fuel depletion and global warming. Especially, as the solar power can be introduced in many places and the maintenance of the solar system is uncomplicated. Introduction of the solar power is rapidly increasing in recent years among the renewable energy.

Geographic Information System (GIS) is known as a tool which can describe the state of ground surface. GIS has continued to steadily develop technology, spatial data, new business and science in many developed countries such as the United States and Europe from 1996 [1]. GIS deals with data referenced by spatial or geographic coordinates [2]. GIS is mainly used in civil engineering. This is reason why considered that GIS is promoted by Land, Infrastructure and Transportation Ministry and Geospatial Information Authority of Japan. For example, GIS is used to manage structures such as roads, tunnels, bridges in the national and local government [3].

This research performs the solar power simulation system by using GIS and Digital Surface Model (DSM).This paper uses this system to grasp and evaluate the locations of solar power introduction. DSM is the elevation data that has height information of buildings and trees. Nearly real 3-D model is made by using DSM [4]. Thus, this research can evaluate a location of the solar radiation amount by considering the shadow of building and trees based on the DSM data [5], [6].

This paper verifies the most efficient angle and the direction of the solar radiation when installing a solar power by using GIS in Kitakyushu city, Fukuoka Prefecture, Japan.

* Manuscript received April 1, 2015; revised August 29, 2015.

Corresponding author. Yuki Koyamatsu Tel.:81-90-5294-0796; E-mail address: k108046y@mail.kyutech.jp.

doi: $10.12720 /$ sgce.4.3.241-246 


\section{Solar Radiation Amount Analysis}

When solar radiation that came from the outer space breaks into the atmosphere, it is affected by the absorption and diffusion with the atmosphere material. In addition, a part of the solar radiation that came from the outer space reflected by the surface of the earth and an atmospheric material is returned in the outer space. The conceptual diagram of the solar radiation balance in the atmosphere is shown in Fig. 1.

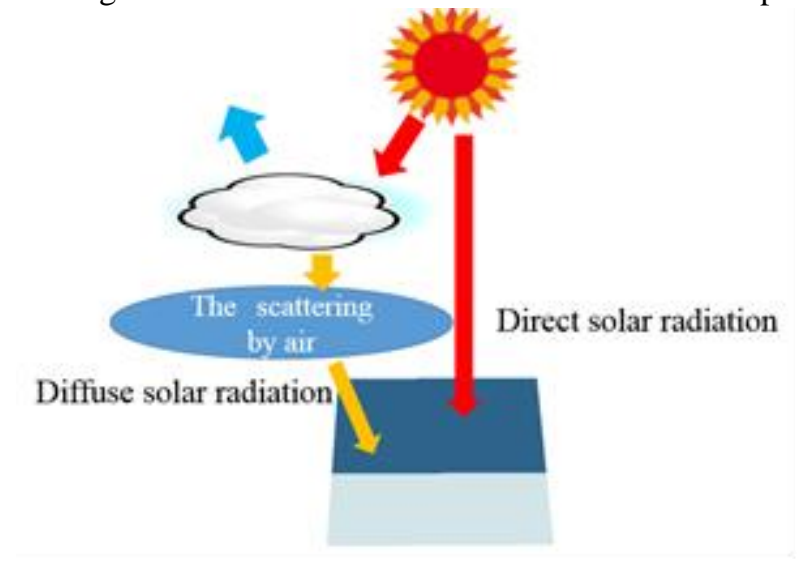

Fig. 1. Solar radiation balance in the atmosphere.

The solar radiation to reach directly the surface of the earth from the sun is called the direct solar radiation [7]. On the other hand, solar radiation reflected by the atmosphere and clouds is called the diffuse solar radiation [7]. All of the solar radiation falling on the surface of the earth is called the global solar radiation. The solar power generation is affected by global solar radiation .The global solar radiation which contains the reflection from the ground surface is shown in equation (1) [8].

\section{$\mathrm{GSR}=\mathrm{DSR} 1+\mathrm{DSR} 2$}

where, GSR is the global solar radiation, DSR1 is the direct solar radiation, DSR2 is the diffuse solar radiation. In general, the global solar radiation is the sum of the direct solar radiation and diffuse solar radiation which contains the reflection from the ground surface as shown in equation (1). The direct solar radiation is the most component in the global solar radiation. Then, the diffuse solar radiation is the second most component in the global solar radiation. The percentage of reflecting solar radiation is small excluding very high reflectivity surface such as snow surface. In other words, the global solar radiation analysis of solar radiation which does not contain the reflection from the ground surface is shown equation (2).

\section{$\mathrm{GSR}=\mathrm{DSR} 1+\mathrm{DSR} 2$}

\section{Analysis of Solar Radiation for the Virtual Surface}

This section shows the way to represent the analysis of solar radiation for the virtual surface.

\subsection{Creating a virtual surface}

This research made a surface with eight directions by using Arc Map of GIS. Each angle of the surface is set 5 degrees of the interval from 0 degrees to 85 degrees. This paper made a virtual surface based on Fig. 2. 


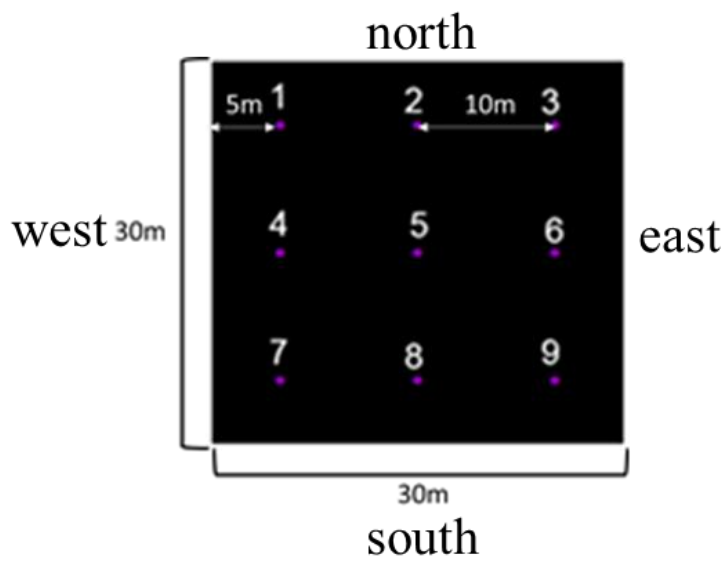

Fig. 2. Virtual surfaces creation (north, south, west and east)

Virtual surfaces were created by entering a value of the height from point 1 to point 9 at Fig. 2. This paper used relative displacement ArcMap's function to create surfaces facing southeast, southwest, northeast and northwest. Fig. 3 shows that the result using a function of relative displacement ArcMap in Fig. 2.

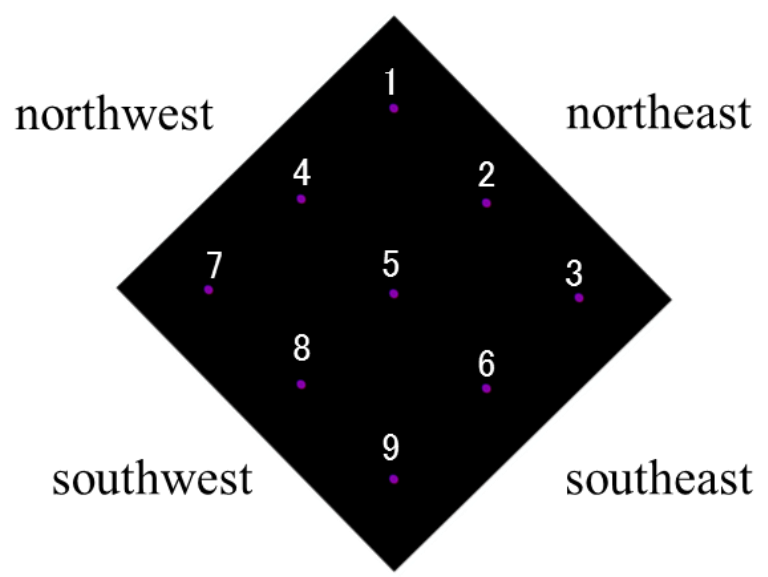

Fig. 3. Virtual surfaces creation (southeast, southwest, northeast and northwest)

Similarly, virtual surfaces were created by entering a value of the height from point 1 to point 9 at Fig. 3. In this process, a virtual surface with eight directions is created. Fig. 4 shows the surface 45 degrees with the east as example.

\subsection{Simulation result}

This paper carried out the analysis of solar radiation to the virtual surface. This paper set parameters as shown below in order to perform the analysis of solar radiation,

- Radiation parameter : UNIFORM_SKY

- Scattering rate : 0.3

- Transparency : 0.5

- Period: One year

UNIFORM_SKY is used in the homogeneous scattering model. The diffuse solar radiation reaches the earth from all directions of the sky and does not change. The scattering rate is the ratio to be scattered in global standard of solar radiation. The range of values is $0 \sim 1$. This value must be set by considering the 
condition of the atmosphere. In general, it becomes 0.3 in the case of sunny. The transparency is the average of the entire wavelength of the amount of solar radiation that passes through the atmosphere. The range of value is $0 \sim 1$. In general, it becomes 0.5 in the case of sunny. First, the above parameters were set to consider the solar radiation only from the solar orbit. Fig. 5 and Fig. 6 shows the results of the solar radiation analysis on the virtual surface.

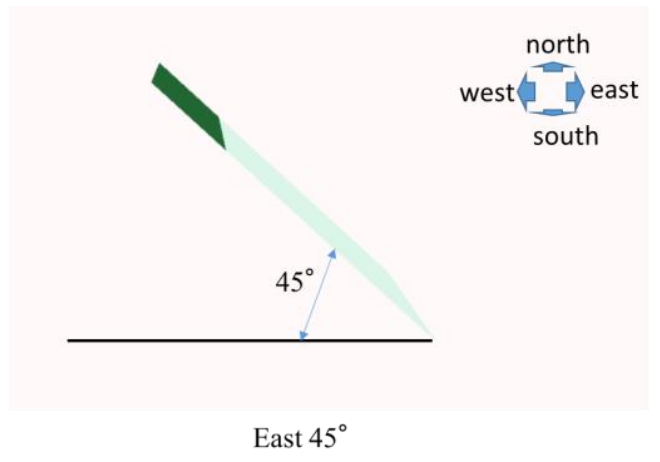

Fig. 4. The surface 45 degrees with the east.

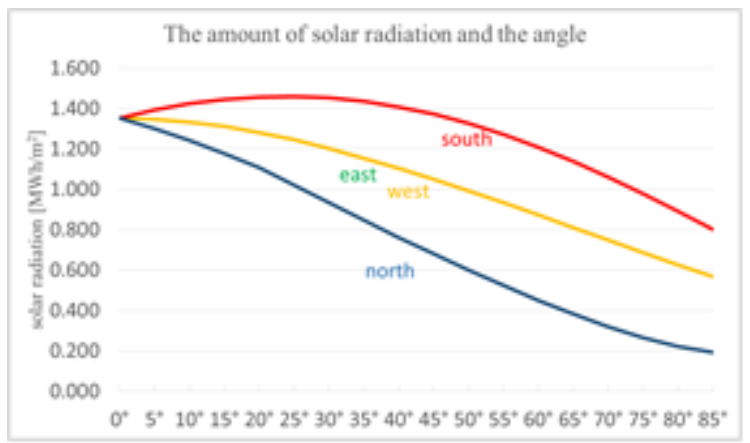

Fig. 5. Angle and direction solar radiation (east, west, south, north).

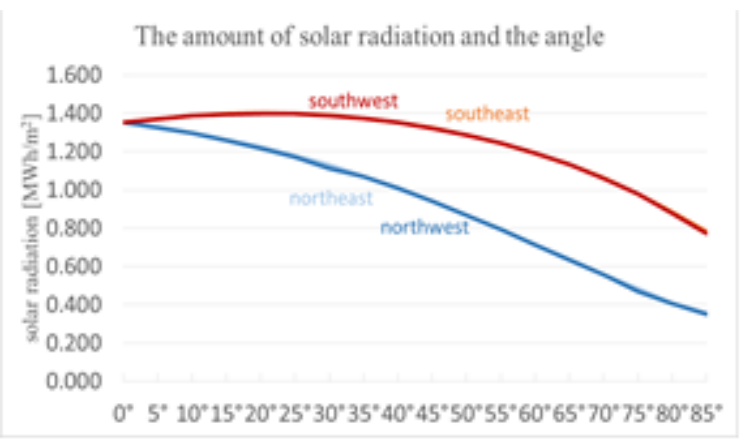

Fig. 6. Angle and direction solar radiation (southeast, southwest, northeast, northwest).

In south, the solar radiation increased from 0 degrees to 25 degrees then it decreased since 25 degrees. In east and west, it increases from 0 degrees to 5 degrees, and it decreases since 5 degrees. In north, it decreases since 0 degrees. In southeast and southwest, they change similarly to the south. In northeast and northwest, it decreases since 0 degrees. Then, Fig. 7 and Fig. 8 shows the percentage of the maximum value is $1.46[\mathrm{MWH} / \mathrm{m} 2]$ at a south-facing to 25 degrees. Fig. 9 shows the figure represented the eight directions. 


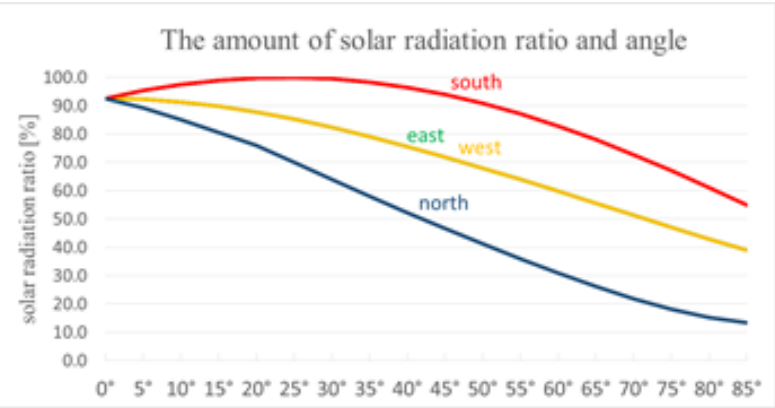

Fig. 7. The amount of solar radiation ratio and the angle and direction (east, west, south, north).

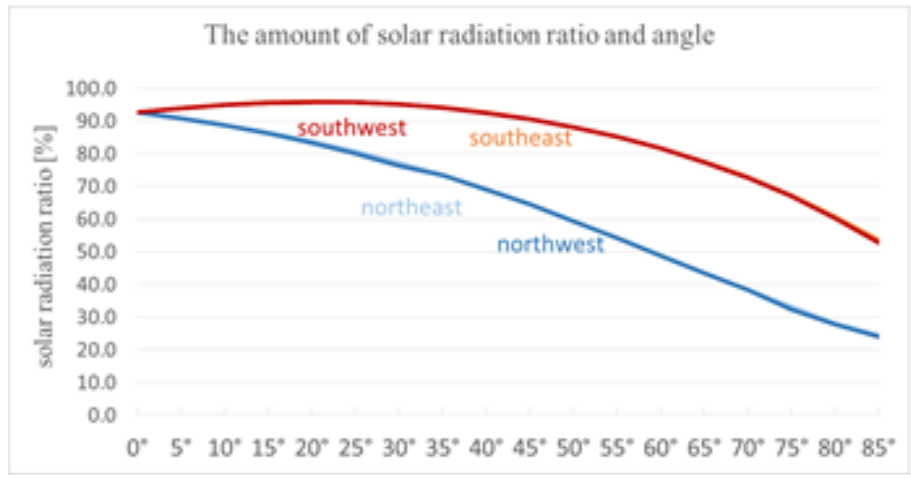

Fig. 8. The amount of solar radiation ratio and the angle and direction (southeast, southwest, northeast, northwest).

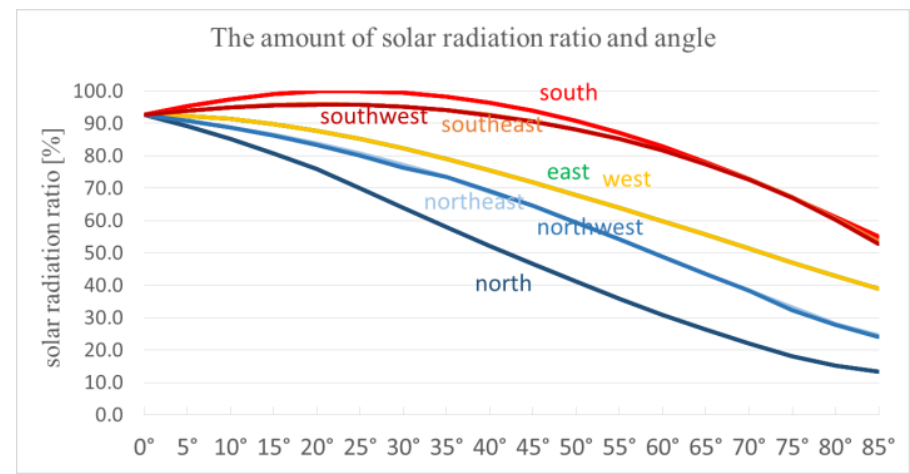

Fig. 9. The amount of solar radiation ratio and the angle and 8 direction.

Fig. 7, Fig. 8 and Fig. 9 show that the angle of the from 0 degrees to 50 degrees ,angle of the southeast and southwest from 0 degrees to 5 degrees,and angle of the east and west from 0 degrees to 15 degrees can obtain $90 \%$ of the maximum solar radiation.

\section{Conclusion}

This research proposes an index for evaluating the amount of the solar radiation of surface facing the 8 direction in Kitakyushu city, Japan. From this result, it was clear that the most efficient angle for the solar power is the south-facing and 25 degrees. Since this paper has created a virtual surface in 5 degrees intervals, more detail analysis between 20 and 30 degrees of facing south is required.

In addition, the relationship between the amount of solar radiation with the angle and the direction is found. If solar panels are unable to be installed for receiving the maximum solar radiation, it is suggested that the other directions in which more over than $90 \%$ maximum of the solar radiation can be gained. 


\section{Acknowledgements}

This research was supported by the G-space City Construction Project of the Ministry of Internal Affairs and Communications at Kitakyushu City of Japan. The authors would like to thank to the research members for their kind assistance.

\section{References}

[1] Hiroyuki K, Atsuyuki O. GIS Sourcebook. Japan: Kokin Shoin; 1996.

[2] Star J, Estes J, Hiroyuki K. Okabe Atsuyuki Geographic Information Systems an Introduction. Japan: Kyoritsu Shuppan Co., Ltd; 1991.

[3] Atsushi S. Utilization of GIS in power system. Master's thesis. Department of Electronic Engineering, Kyushu Institute of Technology, Fukuoka, Japan; 2014.

[4] Lindberg F, Jonsson P, Honjo T, Wastberg D. Solar energy on building envelopes-3D modelling in a 2D environment. Journal of Solar Energy, 2015; 115:369-378.

[5] Shiota A, Fuji K, Kawagoe T, Mitani Y. The outline of the photovoltaic simulation system using GIS. In: Proc. Institute of Electrical Engineers of Japan, 2013:06-1P-09.

[6] Shiota A, Fuji K, Kawagoe T, Mitani Y. System design of the photovoltaic power generation simulator using GIS. In: Proc. The Institute of Electrical Engineers of Japan, 2014:6-099.

[7] Saad N Md, Hamid J R Abdul, Suldi A Mohd. The reliance of insolation pattern on surface aspect. In: Proc. 8th International Symposium of the Digital Earth, 2014:012165

[8] Catita C, Redweik P, Pereira J, Brito MC. Extending solar potential analysis in buildings to vertical facades. Journal of Computers \& Geosciences, 2014; 66:1-12. 